\section{ONOMÁVADIN}

Revista semestral de lingüística, filología y traducción
PONTIFICIA UNIVERSIDAD CATÓLICA DE CHILE CATOLICA DE CHILE
FACULTAD DE LETRAS

\title{
Funciones Discursivas de Consenso y Disenso en Tesis de Lingüística*
}

Discursive Functions of Consensus and

Dissension in Linguistic Theses

\section{Paulina Meza}

Universidad de La Serena

Prodicyt, Centro de Investigación

Chile

\section{Omar Sabaj}

Universidad de La Serena

Prodicyt, Centro de Investigación

Chile

\section{(C) $\underset{B Y}{\text { BY }} \bigodot_{\mathrm{ND}}$}

Paulina Meza: Universidad de La Serena / Prodicyt, Centro de Investigación / Facultad de Ciencias Jurídicas,

Universidad Católica del Norte, Chile. | Correo electrónico: pmeza@userena.cl

Omar Sabaj: Universidad de La Serena / Prodicyt, Centro de Investigación, Chile.

| Correo electrónico: omarsabaj@userena.cl 


\title{
Resumen
}

Para posicionar estratégicamente un objeto de estudio, el autor de una tesis debe equilibrar dos funciones discursivas: por un lado, tiene que reconocer y dar crédito a los referentes teóricos en los cuales su propuesta se enmarca (consenso), y, por otro, para mostrar originalidad, debe diferenciarse de esos trabajos (disenso). A partir del levantamiento de un modelo funcional, analizamos un corpus constituido por 36 Tesis de Lingüística. Los resultados muestran que las funciones de consenso y disenso representan un porcentaje muy exiguo de las tesis $(6,19 \%)$. Asimismo, el consenso se manifiesta en una mayor variedad de funciones y es, por mucho, más frecuente que el disenso. En conclusión, se puede establecer que los resultados se explican por las restricciones que impone el género Tesis. El conocimiento derivado de este trabajo es útil para efectos de la alfabetización académica de estudiantes de educación superior y avanzada.

Palabras clave: consenso; disenso; tesis.

\begin{abstract}
In order to strategically position an object of study, the authors of a thesis must balance two discursive functions: on the one hand, they have to recognize and give credit to the studies that make up the theoretical reference points within which their proposal is framed (consensus/consensus view), and, on the other hand, in order to be original, they must differentiate themselves from those same studies (dissension). Starting from a proposed functional model, we analyze a corpus consisting of 36 linguistics theses. The results show that the functions of consensus and dissension make up a scant percentage of the theses $(6.19 \%)$. Moreover, the consensus view manifests itself in a greater variety of functions and is, by far, much more frequent than dissension. In conclusion, the results can be explained by the restrictions imposed by the thesis genre. The findings of this study have implications for academic literacy in higher education.
\end{abstract}

Keywords: consensus view; dissension; thesis.

\footnotetext{
*Financiamiento: PIA/DIULS 2014. Dirección de Investigación de la Universidad de La Serena.
} 


\section{Introducción}

Una característica central de los géneros académicos, en general, y de la Tesis, en particular, es el hecho de constituir un medio a través del cual el estudiante no solo debe acreditar conocimiento en una determinada área del saber, sino que también debe ser capaz de construirlo. En esta instancia, por tanto, debe ser capaz de glosar los discursos de otro y apropiarse de los modos de decir de la propia disciplina (Arnoux, 2006; Badley, 2009). Esto es sumamente importante si se desea ingresar a alguna comunidad académica, pues, quien pretenda hacerlo, debe ser capaz de comunicar el conocimiento y ha de hacerlo de acuerdo a las normas o cánones establecidos por su disciplina (Meza, 2013).

En este marco, uno de los pilares consustanciales del avance y la comunicación del conocimiento son las nociones de consenso y disenso. El primer concepto lo entendemos como el acto discursivo de concordar, aceptar y legitimar una idea que circula en una comunidad científica. Mientras que el segundo alude, a nuestro juicio, al establecimiento de puntos de distancia, vacíos o problemas en dicha idea. Estas dos fuerzas aparecen plasmadas en los textos mediantes los cuales se comunica el conocimiento, pues son un requisito esencial para ello. En este orden de ideas, la Tesis es un género idóneo para el análisis del consenso y disenso discursivos, pues para comunicar el conocimiento, necesariamente, el tesista debe incorporar en su trabajo, además de la propia, las voces de otros investigadores, y, por tanto, fragmentos donde realice actos de consenso y de disenso. Esta investigación tiene como objetivo identificar las funciones discursivas utilizadas para expresar consenso y disenso en Tesis de Lingüística. Para ello, diseñamos una exploración de corte cualitativo y alcance descriptivo en la que analizamos un corpus compuesto por 36 tesis de lingüística. Combinando métodos deductivos e inductivos, creamos un modelo de análisis propio que permite dar cuenta del consenso y el disenso en el género mencionado. El orden que seguimos en el trabajo es: a) revisión de las nociones de consenso y disenso desde la epistemología y la sociología de la ciencia; b) nuestra propuesta discursiva de consenso y disenso; c) procedimientos metodológicos; d) presentación e interpretación de los resultados; e) principales conclusiones derivadas del trabajo. 


\section{Consenso y disenso: una mirada desde la epistemología y la sociología de la ciencia}

Desde un punto de vista epistemológico, el consenso y el disenso son dos fuerzas consustanciales al desarrollo del conocimiento científico (Ackermann, 1986). En efecto, la idea de consenso equivale a la noción de ciencia normal de Kuhn (1962), mientras que el disenso es, en esos términos, una revolución científica o un cambio de paradigma.

Desde el surgimiento del positivismo hasta los años sesenta, tanto la sociología como la filosofía de la ciencia buscan explicar el impresionante grado de acuerdo en la ciencia (Laudan, 1982). Desde los sesenta en adelante, en cambio, se hace evidente que para explicar el desarrollo científico se debe profundizar en los casos de disenso.

Respecto del estudio del disenso, existen visiones alternativas. Así, Andler (2013) parte del supuesto de que el objetivo natural de cualquier empresa científica es alcanzar el consenso. Asimismo, propone tres etapas que caracterizan el estudio del disenso y reflejan el desarrollo de la historia de la filosofía de la ciencia, a saber: a) el disenso, como accidentes de corta vida, que surge cuando no existe suficiente evidencia que permita decidir qué teoría adoptar (la visión de los padres fundadores); b) el disenso como un estado permanente de la ciencia, en la cual el consenso es un desiderátum inalcanzable (la visión de los hijos díscolos); y c) el disenso como un aspecto esencial, pero transitorio, del desarrollo del conocimiento científico, donde se entiende que el objetivo esencial de la ciencia es el consenso, que es un proceso que se desarrolla de forma extendida, para el cual el disenso es una parte esencial (la visión de los hijos buenos).

Fuller (1986), por su parte, distingue el consenso accidental, el consenso esencial y el consenso esencial sub-óptimo. El primero de ellos se produce cuando dos o más sujetos piensan o hacen lo mismo sin necesidad de dar cuenta públicamente de las razones de su decisión. En el consenso esencial, en cambio, la decisión de hacer o pensar algo se deriva del análisis colectivo y público de las justificaciones individuales, de forma que existe una razón compartida por todos los miembros de un grupo para actuar o pensar de una forma determinada. Por último, el consenso esencial subóptimo refiere al hecho de que no todos los miembros de una comunidad tienen las mismas oportunidades de defender la razón por 
la cual se toma cierta decisión. Específicamente, se critica el ideal del consenso esencial, pues es casi imposible que, cuando se toma una decisión colectiva, todos los miembros puedan exponer sus razones. Si hay ciertas creencias o razones que no son defendidas ni atacadas, se asume erróneamente que todos los miembros de un grupo comparten esa idea, cuando lo que realmente ocurre es que la mayoría se aferra a aquello que los representantes del grupo defienden, pues en cualquier grupo solo hay unos pocos que tienen la vocería. Todos los autores revisados (Andler, 2013; Fuller, 1986; Ackerman, 1986) coinciden en que tratar de determinar los axiomas que definen el estado de consenso en el conocimiento de una disciplina en un momento determinado es una misión, en la práctica, imposible.

\section{Consenso y disenso desde un punto de vista discursivo: el caso de la Tesis de grado}

En este apartado, nos focalizamos en la manifestación discursiva del consenso y el disenso. Desde este punto de vista, Martínez (2008) señala que el estudio del consenso puede contribuir a entender la forma en que los expertos negocian sus puntos de vista y enfoques con otros investigadores. En este sentido, discursivamente hablando, tanto la expresión del consenso como la del disenso corresponden a una actividad dialógica entre dos partes (en este caso, el tesista y otros investigadores).

Knorr-Cetina (1981) reconoce estas dos partes aludidas al señalar que la citación contribuye a la naturaleza dialógica de la escritura del Artículo de Investigación Científica (en adelante, AIC). Así, gran parte del contenido y la argumentación del artículo se escribe con otros autores que también han contribuido al tema; sin embargo, según la autora, esto no se reconoce explícitamente. En consecuencia, los escritores de AIC se basan en las contribuciones de otros autores, pero, al mismo tiempo, los utilizan como una oportunidad para diferenciarse de ellos. Esta es, precisamente, nuestra idea de consenso y disenso discursivos. En relación a este desconocimiento de la escritura con otros a la que alude Knorr-Cetina (1981), Martínez (2008) señala que la omisión de las reivindicaciones se ha asociado con la competencia o con la necesidad de evitar la confrontación. En 
consecuencia, la omisión sería una forma de castigo que contribuiría a la muerte de las afirmaciones de un autor (Myers, 1990).

El consenso y el disenso, necesariamente, deben plasmarse en los textos mediante los cuales se comunica el conocimiento. Gran parte de este conocimiento circula en el ámbito del discurso científico y académico. Así, al escribir estos textos, los autores, obligatoriamente, deben citar otras voces y explicitar si están de acuerdo o no con ellas. Por tanto, con el propósito de adecuarse a las características de estos géneros, los autores deben exponer un conjunto de ideas aceptadas y legitimadas (consenso), pero también, para cumplir con la necesidad de ser originales, deben establecer puntos de distancia, vacíos o problemas en esas ideas mediante la expresión del disenso. En este sentido, un texto académico o científico que solo muestra consenso o que solo da cuenta del disenso no cumple con las condiciones para ser considerado como tal.

En muchas ocasiones, en el momento del descubrimiento, los investigadores no son conscientes de la forma en que su pesquisa se inserta en los debates de su disciplina. Es más bien en el momento de comunicar la investigación en la que se ven en la necesidad de situar el descubrimiento realizado en el contexto de los avances de su campo de especialidad. Es, precisamente, en el contexto de la justificación (Reichenbach, 1938) que los tesistas se ven en la obligación de expresar consenso y disenso.

Como hemos señalado, nos interesa indagar en el consenso y el disenso discursivos en un género específico, que es la Tesis. Optamos por este género debido a su rol fundamental en la difusión y ratificación del conocimiento, así como también en la persuasión a la comunidad académica para aceptar a un nuevo integrante (Koutsantoni, 2006). Este ingreso se conseguiría en la medida en que se conjuguen al mismo tiempo varios factores, como, por ejemplo, el dominio de las estructuras retóricas de los textos que pertenecen a una disciplina y el dominio de la misma (Meza, 2013). Por ello, el tesista debe demostrar suficiencia tanto en sus conocimientos disciplinares como también en las competencias necesarias para investigar y comunicar sus investigaciones (Thompson, 2005; Aleskerov, 2009; Colás y otros., 2009). 
En este género, necesariamente, el tesista debe incorporar las voces de otros investigadores, y, en consecuencia, fragmentos donde realice actos de consenso y de disenso. Según Meza (2013), para comunicar el conocimiento, el tesista acude, entre otros recursos, a diversas fuentes y funciones. La Fuente hace referencia al origen de la información declarada en la Tesis, esto es, el tesista o un autor citado. Así, el tesista utiliza, al menos, dos voces comunicantes: la propia y la de los autores que decide citar, denominadas Fuente Propia y Fuente Ajena, respectivamente (Meza, 2013). Por otro lado, la función se entiende como "la intención con que el autor de la Tesis incorpora ciertos fragmentos en su trabajo" (Meza, 2013). Así, cada fragmento se integra con un motivo concreto y una finalidad que quedará plasmada en el texto.

\section{Métodos}

\subsection{Objetivo y tipo de estudio}

A fin de identificar las funciones discursivas que se utilizan para expresar el consenso y disenso en Tesis de Lingüística, diseñamos una exploración de corte cualitativo (Rodríguez, Gil y García, 1999; Campos, 2009) y de alcance descriptivo (Creswell, 2002, 2007; Vieytes, 2004; Hernández, Fernández y Baptista, 2006).

\subsection{Corpus}

El corpus estuvo compuesto por 36 tesis de Lingüística que cumplían con las siguientes características: a) tres grados académicos (12 de Licenciatura, 12 de Magíster y 12 de Doctorado), b) escritas en español, c) realizadas y aprobadas en universidades chilenas, d) desarrolladas entre los años 2000 a 2009, e) escritas por un solo autor. Además, el corpus cumple con los requerimientos señalados por especialistas: a) Claridad sobre la procedencia (Parodi, 2010), b) Reputación (Nwogu, 1997), c) Integridad (Parodi, 2010).

Una vez conformado el corpus a partir de los criterios descritos, se realizó la división entre un corpus de prueba (33\%) y un corpus de análisis o de investigación (67\%). Esta 
división es necesaria, puesto que las categorías de análisis levantadas necesitan pasar por una etapa de evaluación, que nos permitió ajustarlas y precisarlas.

\subsection{Modelo de análisis discursivo}

Siguiendo los principios de la Teoría Empíricamente Fundada (Strauss y Corbin, 1990, 1997), para el análisis lingüístico combinamos métodos deductivos e inductivos (Ibáñez, 2008; Parodi, 2008, 2010). Así, dejamos que sea cada tesis y su contenido quienes guíen la emergencia de las categorías que permiten describir el fenómeno en estudio.

Para llevar a cabo el análisis, se realizó una lectura detallada y una codificación de los textos del corpus con la ayuda del software Atlas.ti, siguiendo los pasos: a) Identificación de las fuentes o voces, b) Identificación de las funciones, c) Organización del modelo de análisis. Una vez obtenidas todas las categorías de análisis, ellas fueron organizadas, considerando dos criterios: primero, si respondían a un acto de consenso o disenso (filas I, II y III de la tabla 1); y, segundo, de acuerdo a las fuentes involucradas en cada enunciado (columnas A, B y C de la tabla 1).

El análisis realizado pasó por diversos procesos de validación. En cada una de las etapas de análisis (33, 67 y 100\% del corpus) se llevaron a cabo los siguientes procedimientos: a) revisión del análisis, b) triangulación del análisis con otra investigadora, c) exposición del método de análisis a investigadores expertos, d) cálculo del grado de acuerdo entre jueces mediante la aplicación de un instrumento de validación y su análisis estadístico con el coeficiente Kappa de Fleiss. Para el corpus de prueba, el grado de acuerdo entre jueces es de 0,83, lo que, en términos de Landis y Koch (1977), corresponde a un acuerdo casi perfecto; mientras que para el corpus de investigación es de de 0,73 , que, según los mismos autores, es un acuerdo considerable.

Tras la aplicación de todos estos procedimientos, obtuvimos el siguiente modelo. 
Tabla 1. Modelo de análisis: funciones discursivas de consenso y disenso

\begin{tabular}{|c|c|c|c|}
\hline & $\begin{array}{l}\text { A) TESISTA } \rightarrow \text { AUTOR/ } \\
\text { TEORÍA CITADO }\end{array}$ & $\begin{array}{l}\text { B) AUTOR/ TEORÍA } \\
\text { CITADO } \rightarrow \text { TESISTA }\end{array}$ & $\begin{array}{l}\text { C) AUTOR/ TEORÍA } \\
\text { CITADO } 1 \rightarrow \text { AUTOR/ } \\
\text { TEORÍA CITADO } 2\end{array}$ \\
\hline \multirow{4}{*}{$\begin{array}{c}\text { I. } \\
\text { CONSENSO }\end{array}$} & $\begin{array}{l}\text { Indicar acuerdo con los } \\
\text { planteamientos de un autor }\end{array}$ & $\begin{array}{l}\text { Indicar vacíos/ necesidades } \\
\text { en el conocimiento }\end{array}$ & $\begin{array}{l}\text { Indicar coincidencia entre } \\
\text { dos o más autores }\end{array}$ \\
\hline & $\begin{array}{l}\text { Destacar la importancia de } \\
\text { un concepto o teoría }\end{array}$ & Justificar & \\
\hline & $\begin{array}{l}\text { Corroborar resultados de } \\
\text { investigaciones previas }\end{array}$ & Justificar resultados & \\
\hline & Indicar opción teórica & $\begin{array}{l}\text { Citar vacíos/ necesidades } \\
\text { en el conocimiento }\end{array}$ & \\
\hline \multirow{2}{*}{$\begin{array}{c}\text { II. } \\
\text { CONSENSO } \\
\text { Y DISENSO }\end{array}$} & $\begin{array}{l}\text { Indicar aspecto positivo y } \\
\text { negativo de una teoría o } \\
\text { autor citado. }\end{array}$ & & $\begin{array}{l}\text { Indicar un aspecto común y } \\
\text { uno diferenciador entre dos } \\
\text { autores o planteamientos } \\
\text { teóricos }\end{array}$ \\
\hline & $\begin{array}{l}\text { Indicar un aspecto común y } \\
\text { uno diferenciador entre los } \\
\text { hallazgos y los } \\
\text { planteamientos teóricos de } \\
\text { un autor }\end{array}$ & & Reinterpretar propuesta \\
\hline \multirow{2}{*}{$\begin{array}{c}\text { III. } \\
\text { DISENSO }\end{array}$} & $\begin{array}{l}\text { Contradecir resultados de } \\
\text { investigaciones previas }\end{array}$ & & $\begin{array}{l}\text { Indicar desacuerdo entre } \\
\text { dos o más autores }\end{array}$ \\
\hline & $\begin{array}{l}\text { Indicar desacuerdo con los } \\
\text { planteamientos de un autor. }\end{array}$ & & \\
\hline
\end{tabular}

En la primera fila de la tabla, organizamos todas las funciones que dicen relación con el acto discursivo de reconocer y dar crédito a los referentes teóricos en los cuales un autor enmarca su propuesta (consenso). En la tercera fila, consignamos aquellas funciones relacionadas con la necesidad de diferenciarse de otros trabajos (disenso). En la fila intermedia, mostramos las funciones utilizadas para expresar en un mismo enunciado, de forma simultánea el consenso y disenso. 
Por otro lado, en las columnas, se presentan, primero, las funciones cuyos participantes son el tesista y algún autor o teoría que se ha citado. El esquema general en el cual aparecen estos participantes es: Yo (tesista) concuerdo/difiero con X (autor o teoría). En la segunda columna, aparecen las funciones cuyos involucrados también son el tesista y un autor o teoría, pero en este caso, a diferencia del anterior, es el tesista quien pone de su parte al autor citado. El esquema general es: X (autor o teoría) coincide/difiere conmigo (Tesista). En otras palabras, en el caso de la columna A, el tesista es el agente de la acción, mientras que en el caso de la columna B, el agente de la expresión del consenso o disenso es otro autor. Por último, en la tercera columna, aparecen aquellas funciones cuyos participantes son dos autores o teorías, por lo que el consenso o disenso se da entre sujetos distintos del tesista. El esquema general sería: el autor X coincide/difiere con el autor Y.

\section{Resultados y discusión}

\subsection{Cobertura del análisis}

El primer tipo de resultado que presentamos dice relación con la cobertura que logramos con el análisis del corpus, esto es, cuántos enunciados fue posible etiquetar.

Tabla 2. Cobertura del análisis

\begin{tabular}{|l|l|}
\hline $\mathrm{N}^{\circ}$ Total de enunciados & 16.243 \\
\hline $\mathrm{N}^{\circ}$ Enunciados etiquetados & 15.042 \\
\hline$\%$ enunciados etiquetados & $92,61 \%$ \\
\hline $\mathrm{N}^{\circ}$ free quotations & 1.201 \\
\hline$\%$ free quotations & $7,39 \%$ \\
\hline
\end{tabular}

En la tabla 2, presentamos el número total de enunciados que componen el corpus y también la cantidad de fragmentos que no ha sido posible etiquetar (Free quotations). La imposibilidad de etiquetarlos se debió a que la información se presentaba sin que se 
explicitara lingüísticamente si pertenecía al autor de la tesis, si había sido tomada de otro autor o si era el resultado de la interacción entre ambos. Por tanto, no es posible dar cuenta del consenso o disenso. Solo el 7,39\% de los enunciados corresponde a free quotations, por lo que fue posible caracterizar el $92,61 \%$ del total de enunciados del corpus. Con estos datos, podemos afirmar que nuestro análisis presenta una alta cobertura.

\subsection{Consenso y disenso en Tesis de Lingüística}

En la tabla 3, se muestra la frecuencia relativa y absoluta de los segmentos utilizados para expresar: consenso, consenso y disenso en un mismo enunciado y disenso.

Tabla 3. Frecuencia enunciados de consenso y disenso

\begin{tabular}{|l|l|l|l|}
\hline Categoría & $\mathbf{N}$ & $\mathbf{\%}$ & $\begin{array}{l}\text { \% respecto del total de } \\
\text { enunciados etiquetados }\end{array}$ \\
\hline Consenso & 727 & 78,09 & 4,83 \\
\hline Consenso-disenso & 72 & 7,73 & 0,48 \\
\hline Disenso & 132 & 14,18 & 0,88 \\
\hline TOTAL & 931 & 100 & 6,19 \\
\hline
\end{tabular}

Del conjunto de enunciados etiquetados (15.042), solo 931 cumplen alguna función discursiva de consenso o disenso (6,19\%). En este sentido, la proporción de los enunciados destinados a estas funciones, en relación al total de enunciados etiquetados, es bajísima. Estos resultados sugieren que en la Tesis de Lingüística, los autores deben desplegar en su discurso, mayoritariamente, otros propósitos comunicativos requeridos por el género que no dicen relación con la expresión de consenso o disenso. Por ejemplo, aquellos orientados a definir conceptos, describir teorías y señalar aspectos metodológicos (objetivos, procedimientos, definición del tipo de estudio y el alcance, entre otros).

Del total de 931 casos, la mayoría corresponde a enunciados de consenso (78,09\%); luego, en una proporción mucho menor, aparecen los de disenso $(14,18 \%)$; y, por último, 
aquellos enunciados en los que se expresa un acto de consenso y disenso simultáneamente $(7,73 \%)$. Estos resultados pueden ser explicados por las restricciones que impone el género. Específicamente, la Tesis es un género de transición que permite que el tesista vaya integrándose en una comunidad. En esta transición, el tesista debe favorecer su ingreso, señalando que conoce a los referentes de su disciplina, expresando consenso. En este sentido, el nivel de experticia de un tesista hace que sea imposible plantear una idea muy alejada (disenso) de lo ya establecido. Un tesista muy arriesgado que exprese mucho disenso podría fracasar en su ingreso a la comunidad. En términos de Andler (2013), un tesista no puede defender "la visión de los hijos díscolos", vale decir, no puede defender la idea del disenso como un estado permanente de la ciencia. Sobre la base de este resultado, y siguiendo a Collins (1994), las Tesis de Lingüística estarían más cercanas a disciplinas de orden más bien positivista, en las cuales es mucho más fácil alcanzar un estado homogéneo en los conocimientos (consenso). En este sentido, se alejarían de las ciencias sociales, donde, según el autor mencionado, existe gran dificultad para alcanzar el consenso.

Otra interpretación posible para la baja presencia de enunciados de disenso dice relación con la idea de que, en general, la citación es un acto de aserción, en el sentido de que no se cita aquello que parezca irrelevante (Santander, 2002). Por tanto, si se incluye a otro autor en el trabajo es, principalmente, para expresar consenso, para mostrar que es una idea válida. Por el contrario, para evitar la confrontación, las ideas que se consideran erróneas se excluyen, utilizando la omisión como una forma de castigo. Así, la forma de tratar a los autores con los que no se está de acuerdo es ignorarlos (Myers, 1990).

En cuanto a los enunciados que combinan consenso y disenso, su baja presencia puede explicarse por la dificultad que ellos implican. Cuando se escribe una tesis, lo más fácil es concordar con los referentes de un campo disciplinar, puesto que disentir o, más complejo aún, comunicar, a la vez, consenso y disenso, requiere de un conocimiento acabado de ese campo. La expresión simultánea de estas dos funciones se acercaría a "la visión de los hijos buenos" descrita por Andler (2013), en tanto lo que se busca y espera en 
la ciencia es el consenso, que, para ser alcanzado, necesita de alguna forma de disenso. De acuerdo a nuestros datos, esta sería la tarea más difícil de llevar a cabo por los tesistas.

Como ya hemos dicho, es deseable que los tesistas sean capaces de mostrar sus aportes a partir de las investigaciones existentes. Por ello, no resulta extraño que este tipo de enunciados complejos, en los que se realiza consenso y disenso conjuntamente, no sean frecuentes en las tesis analizadas, pues se trata de investigadores en formación. Lo mismo sugiere el hecho de que la expresión de disenso sea poco frecuente: alguien que está tratando de ingresar a una comunidad no puede romper abiertamente con lo establecido.

\subsubsection{Funciones para expresar consenso en Tesis de Lingüística}

En la tabla 4, se muestra la frecuencia absoluta $(\mathrm{N})$ y relativa (\%) de las funciones utilizadas para expresar consenso.

Tabla 4. Frecuencia funciones de consenso

\begin{tabular}{|l|l|l|}
\hline a) Tesista-autor/teoría citado & $\mathbf{N}$ & $\mathbf{\%}$ \\
\hline Indicar acuerdo con los planteamientos de un autor & 25 & 2,69 \\
\hline Corroborar resultados de investigaciones previas & 75 & 8,06 \\
\hline Destacar la importancia de un concepto o teoría & 93 & 9,99 \\
\hline Indicar opción teórica & 292 & 31,36 \\
\hline Subtotal & $\mathbf{4 8 5}$ & $\mathbf{5 2 , 0 9}$ \\
\hline b) Autor/teoría citado- tesista & $\mathbf{N}$ & $\mathbf{\%}$ \\
\hline Citar vacíos/ necesidades en el conocimiento & 16 & 1,72 \\
\hline Justificar & 84 & 9,02 \\
\hline Justificar resultados & 59 & 6,34 \\
\hline Indicar vacíos/ necesidades en el conocimiento & 27 & 2,90 \\
\hline Subtotal & $\mathbf{1 8 6}$ & $\mathbf{1 9 , 9 8}$ \\
\hline c) Autor/teoría citado 1- autor/teoría citado 2 & $\mathbf{N}$ & $\mathbf{\%}$ \\
\hline Indicar coincidencia entre dos o más autores & 56 & 6,02 \\
\hline Subtotal & $\mathbf{5 6}$ & $\mathbf{6 , 0 2}$ \\
\hline Total consenso & $\mathbf{7 2 7}$ & $\mathbf{7 8 , 0 9}$ \\
\hline
\end{tabular}


Para expresar consenso lo más frecuente es que el tesista cite a un autor o teoría (grupo a), esto es, el tesista muestra su acuerdo con determinado autor o planteamiento. Por ejemplo:

(1) Concordamos con Venegas (2005) al afirmar que el texto es una unidad construida en base a la sucesión de unidades lingüísticas coherentes (...).

El segundo caso más frecuente es aquel en que el escritor de la tesis pone a su favor a un autor o teoría (grupo b), vale decir, el tesista señala que cierto autor apoya o coincide con sus planteamientos, como ocurre en el siguiente caso:

(2) La realización del experimento se enfrentó a una serie de dificultades. "El experimento psicológico, que frecuentemente es muy breve en su duración, es fácil de desarrollar en un laboratorio, cuando se tienen sujetos experimentales bien preparados; pero se hace muy complicado en condiciones de trabajo de campo" (Luria, 1987: 29).

En este fragmento, el tesista señala que su experimento tuvo una serie de dificultades y, luego, mediante una cita directa menciona a Luria (1987) para justificar que el experimento psicológico es muy complicado. Así, utiliza a un autor reconocido que, según él, apoya sus planteamientos. De este modo, no deja espacio para que se critique su experimento por las complicaciones, pues un autor muy reconocido lo apoya sobre la complejidad del mismo.

Por último, lo menos frecuente es expresar consenso entre dos autores distintos del tesista (grupo c). Por ejemplo:

(3) A fin de ampliar esta visión del acto de comprender, Peronard (1998) y Johnston (1989) están de acuerdo al señalar que comprender corresponde a establecer conexiones lógicas entre el texto leído y los saberes de mundo propios de cada lector (...).

En este caso, el tesista describe los planteamientos de dos autores que coinciden en su concepción de comprensión. En otros términos, el escritor de la tesis ha sido capaz de reconocer el consenso entre dos importantes investigadores de su área. 
Dentro del primer grupo (a), la función de consenso más frecuente es "Indicar opción teórica" (31,36\%), que refiere a aquellos casos en que el tesista explicita alguna opción tomada:

(4) La perspectiva de análisis que hemos adoptado en esta investigación se denomina enfoque semiodiscursivo (Charaudeau, 2002, 2004, 2005).

Esta función es muy cercana a lo que Fuller (1986) denominaba 'Consenso Esencial' $\mathrm{y}$, en el caso de las tesis analizadas, cumple con el propósito que la audiencia conozca el aparato teórico en el que se enmarca la investigación. Por tanto, es una estrategia que utiliza el tesista para demostrar a sus lectores que sus postulados tienen un anclaje teórico mayor que avala su investigación. En este sentido, esta función es casi obligatoria para desarrollar una tesis, pues, mediante ella, el estudiante evidencia que conoce a los autores importantes de su área, y que es capaz de tomar una decisión entre toda la variedad existente. De este modo, demuestra credibilidad disciplinar, mostrándose a sí mismo como un co-actor que juega un papel en la construcción de conocimiento en su disciplina (Hyland, 2004).

Por otro lado, cuando el autor de la tesis pone al servicio de su investigación a un autor o teoría, buscando y utilizando el consenso a su favor (grupo b), se hace preferentemente con la función "Justificar" $(9,02 \%)$. Esta función incluye tanto las justificaciones sobre lo que hará como también sobre lo que no hará en su investigación. Por ejemplo:

(5) La técnica de evaluación que utilizamos encuentra apoyo en los planteamientos teóricos de Kintsch y van Dijk (1978), van Dijk y Kintsch (1983) y van Dijk (1983) (...).

En el ejemplo, el tesista cita a importantes psicolingüistas, quienes, según él, apoyan la técnica que utilizó en su investigación. Así, le da valor a su trabajo y, en cierto modo, se protege para la instancia de evaluación. Esto, ya que si autores tan renombrados apoyan sus métodos, entonces no cabría una evaluación negativa, pues estaría avalado por sólidos investigadores de su comunidad disciplinar. En este sentido, dicha función se vincula con 
la idea de que la cita podría ser un recurso persuasivo para conferir mayor autoridad a las afirmaciones propias (Gilbert, 1977). Además, esta función coincide con la cita confirmatoria de Kara (2004), que corresponde a las justificaciones de autoridades conocidas para corroborar afirmaciones, resultados, etc. Sin embargo, en nuestra función se destaca el hecho de que es el tesista quien utiliza el consenso a su favor. A nuestro juicio, esta función "Justificar" es un caso curioso porque, en la gran mayoría de los casos, los autores citados ni siquiera conocen la investigación en la que aparecen mencionados y no se enteran de que están propiciando algunas de las ideas allí plasmadas. Sin embargo, los tesistas en su discurso muestran que estos autores apoyan y avalan su trabajo.

Ahora bien, cuando el tesista expresa consenso entre dos autores citados (grupo c), lo realiza mediante una función exclusivamente, a saber, "Indicar coincidencia entre dos o más autores". Un ejemplo es:

(6) Cross (2003), coincide con Livington y Borko (1989) al referirse a la relación entre experticia docente y años de experiencia (...).

Esta función podría relacionarse, por una parte, con lo que Fuller (1986) denomina consenso accidental, en tanto, dos autores pueden coincidir en un mismo juicio pero por razones distintas. Y, por otra, con la idea de cita dialéctica de Kara (2004), que corresponde a aquella que pone a dialogar a varios autores. Así, el tesista no aparece como una voz explícita en la comunicación del conocimiento, sino que solo es el encargado de explicitar la coincidencia entre dos autores.

\subsubsection{Funciones para expresar consenso y disenso en un mismo enunciado}

En este punto, analizamos los casos en los que consenso y disenso se expresan conjuntamente en un mismo enunciado. 
Tabla 5. Funciones para expresar conjuntamente consenso y disenso

\begin{tabular}{|l|l|l|}
\hline a) Tesista-autor/teoría citado & $\mathbf{N}$ & $\mathbf{\%}$ \\
\hline $\begin{array}{l}\text { Indicar un aspecto común y uno diferenciador entre los hallazgos propios y los } \\
\text { planteamientos teóricos de un autor }\end{array}$ & 14 & 1,5 \\
\hline Indicar aspecto positivo y negativo de una teoría o autor citado & 9 & 0,97 \\
\hline Subtotal & 23 & 2,47 \\
\hline b) Autor/teoría citado- tesista & $\mathbf{N}$ & $\mathbf{\%}$ \\
\hline Subtotal & $\mathbf{0}$ & $\mathbf{0}$ \\
\hline c) Autor/teoría citado 1- autor/teoría citado 2 & $\mathbf{N}$ & $\mathbf{\%}$ \\
\hline $\begin{array}{l}\text { Indicar un aspecto común y uno diferenciador entre dos autores o } \\
\text { planteamientos teóricos }\end{array}$ & 16 & 1,72 \\
\hline Reinterpretar propuesta & 33 & 3,54 \\
\hline Subtotal & 49 & 5,26 \\
\hline Total consenso-disenso & 72 & 7,73 \\
\hline
\end{tabular}

Como aparece en la tabla 5, estas funciones, que podemos denominar complejas por el hecho de que el consenso y el disenso se expresan en un mismo fragmento, son las menos frecuentes. Es interesante notar que estos enunciados solo aparecen cuando es el tesista quien propicia la expresión del consenso y el disenso (grupo a), o bien cuando el tesista señala consenso y disenso entre dos autores (grupo c), pero nunca cuando es el tesista quien pone a un autor de su parte (grupo b).

Con respecto al grupo c, esto es, cuando consenso y disenso aparecen en un mismo enunciado y los participantes son otros autores o teorías distintos del tesista, este se expresa principalmente mediante la función "Reinterpretar propuesta teórica". En estos casos, la voz del tesista no aparece de forma directa, sino que su función es orquestar las voces de otros autores. Así, el tesista describe los cambios que un autor hizo a la propuesta de otro, tal como en el siguiente ejemplo: 
(7) Marinkovich, Peronard \& Parodi (2006) recogen la distinción propuesta por Rosenblatt (1978) entre la lectura eferente, la que ellos llaman alternativamente lectura de estudio o "lectura para aprender", y la lectura estética o "lectura para pasar el rato"; y agregan una tercera modalidad, la lectura funcional (...).

La segunda función del grupo c, denominada "Indicar un aspecto común y uno diferenciador entre dos autores o planteamientos teóricos", se utiliza para señalar aquellos casos en que un autor $\mathrm{X}$ tiene un punto en común (consenso) y uno en desacuerdo (disenso) con los planteamientos de otro autor, por ejemplo:

(8) A nuestro parecer, tanto la propuesta de Ciapuscio (2001) como la de Cassany (2001) tienen perspectivas convergentes y complementarias. Por ejemplo, la estrategia de expansión, tiene que ver con la incorporación de contenidos que puede ser de naturaleza informativa o emotiva. Esta incorporación del aspecto emotivo que hace Ciapuscio (2001), no está presente en el modelo de Cassany (2001) (...).

En estos casos, aun cuando su voz no aparezca directamente, al realizar un acto de consenso y disenso en un mismo enunciado, el tesista muestra dominio disciplinar del tema, pues es capaz de reconocer aspectos comunes y diferenciadores entre distintos autores, evidenciando, así, su conocimiento teórico y bibliográfico. Esto no solo implica una presentación de la información de otros, sino también una transformación de ella en conocimiento nuevo.

Por otra parte, cuando los participantes de la expresión del consenso y el disenso en un mismo enunciado es propiciada por el tesista, utilizando como referente a un autor o teoría (grupo a), se realiza, preferentemente, con la función "Indicar un aspecto común y uno diferenciador entre los hallazgos propios y los planteamientos teóricos de un autor". Esta función aparece cuando los hallazgos propios son confirmados parcialmente por otro autor. Generalmente, con esta función el tesista busca interpretaciones para explicar por qué sucede esto, indicando, a menudo, que se debe a diferencias metodológicas. Un ejemplo de esta función es: 
(9) En cuanto a la correlación /p-t-k/ vs. /b-d-g/ todos los estudios encontraron una proporción más o menos similar en los pares mínimos cuyas realizaciones se articulan en la zona anterior (...) La diferencia más significativa se manifiesta entre $/ \mathrm{k} / \mathrm{y} / \mathrm{g} /(4.02 \%$ y $1.02 \%$ respectivamente), hecho que coloca a la consonante de realización velar áfona en el tercer lugar en términos de frecuencia dentro de la correlación, superando incluso a /d/ en el estudio de Guirao y García Jurado.

La otra función que aparece entre estos participantes del grupo a es de carácter teórico, a saber, "Indicar aspecto positivo y negativo de una teoría o autor citado". El tesista, para posicionar su propia investigación, se ve en la necesidad de, por un lado, señalar aquellos aspectos que, desde un punto de vista teórico, comparte con otros referentes de su campo disciplinar; y, por otra, referir a aquellos aspectos que no comparte. Estos recursos retóricos, que muestran un vaivén entre el consenso y el disenso, son esenciales para el ejercicio académico, especialmente, cuando se trata de producir un género como la Tesis, que le permite al estudiante avanzar en su formación académica o ingresar a la comunidad científica de su disciplina. Por ejemplo:

(10) El trabajo de Bucks y colegas (2000) resulta ser un aporte importante para diferenciar el habla de sujetos que padecen una demencia de un grupo adultos mayores sanos. Sin embargo, en términos de las interpretaciones para explicar el funcionamiento de la memoria, resulta demasiado simple (...).

\subsubsection{Funciones para expresar disenso en Tesis de Lingüística}

En la tabla 6, aparece la frecuencia de las funciones utilizadas para expresar disenso.

Tabla 6. Funciones para expresar disenso

\begin{tabular}{|l|l|l|}
\hline a) Tesista-autor/teoría citado & $\mathbf{N}$ & $\mathbf{\%}$ \\
\hline Contradecir resultados de investigaciones previas & 47 & 5,05 \\
\hline Indicar desacuerdo con los planteamientos de un autor & 15 & 1,61 \\
\hline Subtotal & 62 & 6,66 \\
\hline b) Autor/teoría citado- tesista & $\mathbf{N}$ & $\mathbf{\%}$ \\
\hline
\end{tabular}




\begin{tabular}{|l|l|l|}
\hline Subtotal & $\mathbf{0}$ & $\mathbf{0}$ \\
\hline c) Autor/teoría citado 1- autor/teoría citado 2 & $\mathbf{N}$ & $\mathbf{\%}$ \\
\hline Indicar desacuerdo entre dos o más autores & 70 & 7,52 \\
\hline Subtotal & 70 & 7,52 \\
\hline Total disenso & 132 & 14,18 \\
\hline
\end{tabular}

La función más frecuente para expresar disenso surge cuando los participantes son otros autores (c), específicamente, con la función "Indicar desacuerdo entre dos o más autores", utilizada para posicionar la propia investigación sin involucrarse directamente en la expresión del disenso. A través de la citación de dos o más autores, el tesista indica desacuerdo entre ellos, o entre el mismo autor en dos investigaciones diferentes. Por ejemplo:

(11) Las antiguas teorías conductistas se contraponen taxativamente con la hipótesis innatista encabezada por Chomsky (citado en López Ornat, 1999) y, parcialmente, con el enfoque cognitivista propuesto por Piaget (citado en Crystal, 1987) debido a que ambos atribuyen el aprendizaje al ambiente que rodea al niño.

Esta función podría equipararse con la idea de cita dialéctica de Kara (2004), mencionada anteriormente. Sin embargo, nuestro nivel de descripción es más preciso, puesto que especificamos el motivo por el cual se ponen en diálogo distintos autores.

Por otra parte, cuando es el tesista quien propicia el disenso, usando como referente a un autor o una teoría (grupo a), lo realiza con dos funciones: "Contradecir resultados de investigaciones previas" e "Indicar desacuerdo con los planteamientos de un autor". En términos estratégicos, es interesante señalar que estas son las únicas dos maneras que utilizan los tesistas para diferenciarse del resto de las investigaciones realizadas en su campo. Tal como indican Myers (1990), Martínez (2008) y Knorr-Cetina (1981), una forma de evitar la confrontación es simplemente no citar a autores con los que no se está de acuerdo. Esto podría explicar por qué la frecuencia de las funciones del disenso es tan baja 
y poco variada. Por un lado, un ejemplo para "Contradecir resultados de investigaciones previas" es:

(12) En este sentido, nuestros resultados son diferentes de los hallados por Blanken et al. (1987), pues al analizar el habla de la conversación en pacientes con EA, afásicos de Wernicke y un grupo control, los autores no encontraron que los sujetos con EA presentaran más problemas para hallar palabras que los adultos mayores normales.

En este caso, a partir de los resultados de su investigación, el tesista muestra la discordancia existente entre sus hallazgos y los resultados de investigaciones previas de otros autores. Con respecto a esta función, debemos recordar que Massi (2005) señala que una de las tres funciones de las citas es establecer relaciones entre un texto existente y el que está en elaboración, a fin de comentar, asentir, disentir o señalar incongruencias. Esta afirmación podría vincularse con la función "Contradecir resultados de investigaciones previas”. Sin embargo, el desarrollo del punto de Massi (2005) es muy general y, además, solo lo ve desde la citación, y no desde el vínculo con la inscripción de la persona. Así mismo, la presencia de dicha función en nuestro corpus nos permite validar solo parcialmente la afirmación de Hyland (1999), que señala que la citación es un procedimiento de ratificación de lo dicho por el escritor, puesto que, como vemos a partir de muchas de las funciones aquí planteadas, no solo se cita para justificar.

Por otro lado, la función "Indicar desacuerdo con los planteamientos de un autor" se utiliza para explicitar que el tesista no está de acuerdo, en algún punto, con un autor determinado. Para realizar esta función, a diferencia de lo que ocurría con "Contradecir resultados de investigaciones previas", el tesista no tiene la evidencia empírica de sus hallazgos para manifestar su desacuerdo, sino que es un disenso que está más bien al nivel de la opinión. No sería, en este sentido, una creencia justificada con datos, sino que su único respaldo es el ethos del tesista. Por ejemplo:

(13) Volvamos, por ejemplo, a la definición que se otorgaba para el verbo (cfr. RAE, 1973/ 2000; Pérez-Rioja, 1971). Claramente, el hecho de que el verbo se defina como aquella 
parte de la oración que expresa acción, pasión o estado es altamente reduccionista ya que existen otras categorías que también cumplen con estas funciones. Así por ejemplo, el adjetivo se traslapa con el verbo en cuanto ambos son unidades predicativas. Además por otra parte, aunque el verbo está prototípicamente ligado a la acción, a la pasión y al estado, en estos últimos dos casos, también otras categorías pueden cumplir esa función, a saber, los adjetivos y los participios.

En síntesis, podemos señalar, entonces, que lo más típico en las Tesis de Lingüística es la expresión del consenso, seguido por el disenso y, por último, de la expresión de ambos en un mismo enunciado (tabla 4). Esto, como ya lo hemos señalado, podría deberse, por un lado, a la preferencia por la omisión antes que la confrontación (Knorr-Cetina, 1981; Myers, 1990; Martínez, 2008); y, por otro, a la dificultad que implica conjugar los actos de consenso y disenso en un mismo enunciado. Así también, es interesante notar que cuando se expresa disenso la variabilidad de las funciones es menor (3) que cuando se expresa consenso (9). Por su parte, la expresión de consenso y disenso en un mismo enunciado tiene una variabilidad intermedia en relación a las otras (4).

Resulta interesante notar que el esquema de participantes del grupo b (el tesista utiliza un autor para apoyar su investigación) solo se realiza para expresar consenso. Esto es, nunca citaré a quién me contradiga, aunque yo puedo contradecir (y también estar de acuerdo con) a quienes cito; vale decir, cuando el tesista utiliza a otros autores para apoyarse solo lo hace cuando esos autores están de acuerdo con él. En este sentido, el esquema que aparece es "Tales autores apoyan mi idea" y no "tales autores no apoyan mi investigación" o "tales autores apoyan esta parte de mi idea pero no esta otra". En cambio, si se trata de expresar solo disenso o disenso y consenso en un mismo enunciado, siempre es el tesista el agente de la función, lo que en términos esquemáticos equivale a "Yo no estoy de acuerdo con tal autor" o "Yo estoy de acuerdo con esta parte de la teoría del autor y en desacuerdo con esta otra". 


\section{Conclusiones}

A partir del análisis que dio origen a la propuesta, advertimos que para expresar consenso y disenso en el género Tesis, se recurre a dos voces o Fuentes principales: el tesista y los autores citados, quienes interactúan constantemente para realizar los actos de consenso y disenso. Sin embargo, en algunos casos, el tesista se incorpora solo para mostrar cómo otros autores concuerdan o difieren en alguna idea, orquestando sus voces. Del mismo modo, encontramos que los actos de consenso y disenso no siempre se presentan de manera independiente, sino que, en ocasiones, estos dos actos discursivos constituyen un mismo enunciado.

En síntesis, como conclusiones centrales de esta investigación, se puede establecer que:

a) Los enunciados para expresar consenso o disenso representan solo un $6,19 \%$ del total del corpus.

b) Lo más común es expresar consenso y lo menos frecuente es comunicar de forma conjunta consenso y disenso en un mismo enunciado.

c) Para expresar consenso existe una mayor variabilidad de funciones (9) que para la comunicación del disenso (4).

d) Lo más común es que sea el tesista quien se presenta como el agente de la expresión de consenso o disenso (grupo a) y lo menos frecuente es que el tesista utilice a un autor o teoría como el agente de dichas funciones (grupo b).

Destacamos como aporte de nuestro trabajo la creación, a partir de un análisis basado en corpus, de una propuesta de análisis bastante exhaustiva para el consenso y el disenso, que no había sido planteada previamente. Esta propuesta consta de 3 grupos de actores (tesista y autor o teoría citado, autor o teoría citado y tesista, autor o teoría citado 1 y autor o teoría citado 2), 3 tipos de enunciados (consenso, disenso, y consenso-disenso) y 16 funciones para expresar estos tipos de enunciados (ver tabla 1). Creemos que la 
investigación es un aporte para entender cómo diversas fuentes o voces interactúan en los textos para mostrar consenso o disenso.

Estos hallazgos constituyen un material valioso para ayudar a estudiantes de distintos grados académicos en su proceso de escritura de Tesis, ya que les permite conocer la utilización estratégica de las funciones de consenso y disenso, asociadas a cada fuente de la información. Así, nuestra investigación puede ayudarlos a saber con certeza cuáles son las formas existentes para dar cuenta de aquellos casos en que se está de acuerdo con un autor y también en los que se difiere con ellos.

\section{Bibliografía citada}

Ackermann, Robert. 1986: "Consensus and Dissensus in Science", Proceedings of the Biennial Meeting of the Philosophy of Science 1986, 99-105.

Aleskerov, Fuad, 2009: "How to prepare and write PhD and MS thesis", Automation and Remote Control 70, 1928-1938.

ANDLER, Daniel, 2013: "Dissensus in Science as a Fact and as a Norm", The Philosophy of Science in a European Perspective 4, 493-506.

ARnOUX, Elvira, 2006: "Incidencia de la lectura de pares y expertos en la reescritura de tramos del trabajo de Tesis", Revista de Lingüística Teórica y Aplicada 44(1), 95-118.

BADLEY, Graham, 2009: "Academic writing as shaping and re-shaping", Teaching in Higher Education 14(2), 209-219.

CAmpos, Agustín, 2009: Métodos mixtos de investigación, Bogotá: Magisterio.

ColÁs, María Pilar, Leonor Buendía y Fuensanta Hernández, 2009: Competencias científicas para la realización de una Tesis doctoral, Barcelona: Da Vinci.

Collins, Randall, 1994: "Why the Social Sciences Won't Become High-Consensus, Rapid-Discovery Science”, Sociological Forum 9(2), 155-177.

CRESWELL, John, W. 2002: Research design. Qualitative, quantitative and mixed methods approaches, Thousand Oaks: Sage Publications. 
CRESWELl, John, 2007: Qualitative Inquiry \& Research design. Choosing among five approaches, Thousand Oaks: Sage Publications.

FUller, Steve, 1986: “The Elusiveness of Consensus in Science”, Proceedings of the Biennial Meeting of the Philosophy of Science Association, 106-119.

GILBERT, Nigel, 1977: “Referencing as persuasion”, Social Studies of Science 7, 113-122.

Hernández, Roberto, FERnÁndez y Pilar BAPtista, 2006: Metodología de la investigación, México: McGraw-Hill.

HyLAND, Ken, 1999: "Academic attribution: Citation and the construction of disciplinary knowledge", Applied Linguistics 20 (3), 341-367.

Hyland, Ken, 2004: Disciplinary discourses, Michigan: The University of Michigan Prless.

IBÁÑEZ, Romualdo, 2008: "El texto disciplinar y el acceso al conocimiento desde el análisis del género: ¿Regulación del conocimiento o persuasión?” en Giovanni PARODI (ed.): Géneros académicos y géneros profesionales: accesos discursivos para saber y hacer, Valparaíso: Ediciones Universitarias de Valparaíso, 219-246.

KARA, Mohamed, 2004: "Pratiques de la citation dans les mémoires de maîtrise", Pratiques: Théorie, pratique, pédagogie 121/122, 111-142.

KnORR-CETINA, Karin, D. 1981: The manufacture of knowledge: An essay on the constructivist and contextual nature of science, Oxford: Pergamon.

KoutsantONI, Dimitra, 2006: "Rhetorical strategies in engineering research articles and research theses: Advanced academic literacy and relations of power", Journal of English for Academic Purposes 5 (1), 19-36.

KuHn, Thomas, 1962: The Structure of Scientific Revolutions, London: The University of Chicago Press.

LAUDAN, Larry, 1982: "Two puzzles about science: Reflections on some crises in the philosophy and sociology of science”, Minerva 20(3-4), 253-268.

LANDIS, John y Gary KoCH, 1977: “The measurement of observer agreement for categorical data", Biometrics 33, 159-174. 
MARTÍNEZ, Iliana, 2008: "Building consensus in science: Resources for intertextual dialog in biology research articles”, Journal of English for Academic Purposes 7, 268- 276.

MASSI, María, 2005: Las citas en la comunicación académica escrita [http://www.rieoei.org/deloslectores/1011Palmira.PDF, fecha de consulta: 27 de abril de 2012].

MezA, Paulina, 2013: La comunicación del conocimiento en las secciones de Tesis de Lingüística: determinación de la variación entre grados académicos. Tesis doctoral, Pontificia Universidad Católica de Valparaíso, Valparaíso, Chile.

MYERS, Greg, 1990: Writing biology, London: The University of Wisconsin Press.

NwoGU, Kevin, 1997: “The medical research paper: structure and functions”, English for Specific Purposes 16 (2), 119-138.

PARODI, Giovanni, 2008: “Géneros académicos y géneros profesionales: Delimitaciones y puntos de encuentro en el Corpus PUCV- 2006” en Giovanni PARODI (ed.): Géneros Académicos y Géneros Profesionales: Accesos discursivos para Saber y Hacer, Valparaíso: Ediciones Universitarias de Valparaíso, 75-96.

PARODI, Giovanni, 2010: Lingüística de corpus: de la teoría a la empiria, Madrid: Iberoamericana Vervuert.

ReICHEnbach, Hans, 1938: Experience and Prediction: An analysis of the Foundations and the Structure of Knowledge, Chicago: University of Chicago Press.

RodríGUEZ, Gregorio, Javier GIL y Eduardo GARcíA, 1999: Metodología de la investigación cualitativa, Málaga: Aljibe.

SABAJ, Omar y Denisse LandeA, 2012: "Descripción de las formas de justificación de los objetivos en artículos de investigación en español de seis áreas científicas”, Onomázein 25 (1), 315-344.

SANTANDER, Pedro, 2002: El acceso como mecanismo de control discursivo en el género informativo de la televisión chilena. Tesis doctoral, Pontificia Universidad Católica de Valparaíso, Valparaíso.

STRAUSS, Anselm y Juliet CORBIN, 1990: Basics of qualitative research: grounded theory procedures and techniques, London: Sage. 
ONOMÁZEIN 33 (junio de 2016): 385-411

Paulina Meza y Omar Sabaj:

Funciones discursivas de consenso y disenso en tesis de lingüística

STRAUSS, Anselm and Juliet CORBIN (eds). 1997: Grounded theory in practice, London: Sage.

ThOMPSON, Paul, 2005: "Points of focus and position: Intertextual reference in $\mathrm{PhD}$ theses", Journal of English for Academic Purposes 4, 307-323.

VIEYTES, Rut, 2004: Metodología de la investigación en organizaciones, mercado y sociedad. Epistemología y técnicas, Buenos Aires: Editorial de las Ciencias. 